\title{
Surgical treatment of non-cystic fibrosis bronchiectasis in Central Europe
}

\author{
Alaa Selman", Hayan Merhej", Tomoyuki Nakagiri, Norman Zinne, Tobias Goecke, Axel Haverich, \\ Patrick Zardo
}

Department of Cardiothoracic, Transplantation and Vascular Surgery, Hannover Medical School, Hannover, Germany

Contributions: (I) Conception and design: A Selman, H Merhej, P Zardo; (II) Administrative support: N Zinne, T Goecke; (III) Provision of study materials or patients: A Haverich, P Zardo; (IV) Collection and assembly of data: A Selman, H Merhej; (V) Data analysis and interpretation: A Selman, H Merhej, T Nakagiri, P Zardo; (VI) Manuscript writing: All authors; (VII) Final approval of manuscript: All authors.

\#These authors contributed equally to this work.

Correspondence to: Patrick Zardo. Department of Cardiothoracic, Transplantation and Vascular Surgery, Hannover Medical School, Carl-NeubergStraße 1, 30625 Hannover, Germany. Email: zardo.patrick@mh-hannover.de.

Background: Bronchiectasis is a mostly irreversible bronchial dilatation induced by the destruction of elastic and muscular fibers of the bronchial wall. Surgical treatment is usually reserved for focal disease, and whenever complications, like hemoptysis or secondary aspergilloma, arise. In this study, we report our experience and outcomes in surgical bronchiectasis management between 2016 and 2020.

Methods: We retrospectively searched our database for patients admitted for surgical treatment of bronchiectasis between 2016 and 2020. All records were screened for pre-surgical management. Age, gender, distribution of bronchiectatic lesions, type of surgery, perioperative complications, chest tube duration, length of hospital stay as well as 30-day-mortality were recorded, and a brief follow-up was made.

Results: A total of $\mathrm{n}=34$ patients underwent pulmonary resection with bronchiectasis. Mean age on admission was $56.2 \pm 15.1$ years and $n=21$ patients $(62 \%)$ were female. In $n=23$ cases the right lung was affected, in $\mathrm{n}=9$ cases the left side and in two cases both lungs. Indications for surgery included persistent major alterations after conservative therapy $(n=9)$, massive hemoptysis $(n=4)$, and full-blown "destroyed lobe" $(n=7)$. All patients received anatomical lung resection $(n=21$ lobectomies, $n=2$ bilobectomies and $n=11$ segmentectomies), either by uniportal video assisted thoracoscopic surgery $(n=28)$ or by lateral thoracotomy $(n=6)$. Average length of hospital stay was $7.9 \pm 6.3$ days; one patient died on POD 7 due to myocardial infarction.

Conclusions: In spite of a decreasing number of patients with bronchiectasis referred to surgery due to improvements in preventing and managing the disease, pulmonary resection still plays a significant role in treating this pathology in Central Europe. Surgery remains a viable approach for localized forms of bronchiectasis, and the only option in treating acute deterioration and complications like massive hemoptysis.

Keywords: Bronchiectasis; non-CF-bronchiectasis; hemoptysis; surgical treatment; uniportal video-assisted thoracoscopic surgery (VATS) resection

Submitted May 26, 2021. Accepted for publication Aug 26, 2021.

doi: $10.21037 /$ jtd-21-879

View this article at: https://dx.doi.org/10.21037/jtd-21-879 


\section{Introduction}

Bronchiectasis is a mostly irreversible bronchial dilatation induced by destruction of elastic and muscular fibers of the bronchial wall. Radiological criteria of bronchiectasis are met, when the inner diameter of the bronchial wall surpasses the outer diameter of the accompanying pulmonary artery (signet ring sign) $(1,2)$. Its incidence increases with age, even though it often lacks true clinical signs of disease. Only when it is accompanied by cough, expectorations, and recurring bronchopulmonary infections, it can be considered a true bronchiectatic disease. Cystic fibrosis (CF) is one of its preeminent triggers, but certainly plays a particular role in this entity, which is why the terminus of "non-CF-Bronchiectasis" was coined in the first place (3).

A congenital form of non-CF-bronchiectasis has been observed $(4,5)$, but acquired variations are far more abundant. Repeated respiratory tract infections, especially by tuberculosis, measles or pertussis, are the main cause for developing bronchiectasis, even though bronchial obstruction by tumor or foreign body aspiration may play a role. Additionally, bronchiectasis has been seen in allergic bronchopulmonary aspergillosis, primary ciliary dyskinesia, $\alpha 1$-antitrypsin deficiency, congenital and acquired immunodeficiency states, intralobar or extralobar sequestrations, sarcoidosis and non-tuberculous mycobacteria (NTM)-Infection (2-5). Chronic obstructive pulmonary disease (COPD) patients showing evidence of bronchiectasis are known to often present with more severe symptoms, a higher frequency of exacerbation, lower lung function and increased mortality (6). Despite an association having been recognized, no true causal relationship for a COPD-Bronchiectasis-OverlapSyndrome is known to exist (7), even though patients may present with both entities at once.

Even though there is a general belief that its global incidence has declined due to overall improvements in healthcare delivery and systematic, large-scale vaccination programs during the past decades, especially in developed countries, recent publications confirm that this pathology remains largely unknown or underestimated (8). Documented incidences vary widely (67-362/100,000 adults) between different countries and reporting groups, most likely in accordance with availability of diagnostic tools $(9,10)$.

A national population-based cohort study in Germany even concludes that the number of patients affected by bronchiectasis increases at a yearly rate of $10 \%$, and stresses the need for specialized multidisciplinary teams (MDT) to address the issue (11).

Localization and manifestation of bronchiectasis is closely related to the underlying disease: The left lower lobe and lingula are usually affected by postinfectious forms, whereas tuberculosis usually manifests itself in the right upper lobe (12). Whole lung bronchiectasis is more likely to be found on the left side (13). Middle lobe affliction can be observed in middle lobe syndrome or tuberculosis (14).

Optimal treatment is complex, often primarily symptomatic, multifactorial, and should be made by a MDT (3). Overall strategy is mandated by localization and underlying disease, with factors like severity of symptoms, frequency of complications and the patient's respiratory capacity playing a role as well. Initial management usually comprises a combination of antibiotic therapy, secretolytic and physiotherapy, cessation of smoking and vaccination (3). Before opting for a surgical approach, this protocol should be repeated at least three times and continued for at least 2 years unless severe complications like major hemoptysis occur.

Nowadays a surgical approach is being reserved for focal disease, whenever medical treatment fails, and severe complications such as massive hemoptysis, recurrent pneumonia or secondary colonization with aspergillus occur. In these cases, treatment consists in resection of affected parenchyma, usually in form of anatomical lung resection (15).

The purpose of our study was to analyze cases of bronchiectasis warranting surgical resection in a tertiary treatment setting in Central Europe. We describe its clinical presentation, causes, employed diagnostic methods and indication for surgery. Additionally, we evaluated our surgical approach to determine feasibility and safety of minimally invasive surgery in bronchiectasis.

We present the following article in accordance with the STROBE reporting checklist (available at https://dx.doi. org/10.21037/jtd-21-879).

\section{Methods}

The study was conducted in accordance with the Declaration of Helsinki (as revised in 2013). The study was approved by institutional ethics board of the Hannover Medical School (NO.: 9789_B0_K_2021) and individual consent for this retrospective analysis was waived. After obtaining internal review board approval, we searched our institutional database for patients receiving surgical 
treatment for bronchiectasis between 01.01.201631.12.2020. Age, gender, pre-surgical duration of symptoms, radiological distribution of disease, type and duration of procedure, intraoperative/postoperative complications, postoperative chest tube duration, post-procedural duration of analgesic therapy, length of hospital stay as well as mortality and morbidity were recorded.

Follow-up information was obtained by telephonic interview at least half a year after surgery. Clinical outcome of surgery was evaluated by comparing current and preoperative performance status. This was classified as either excellent (complete absence of perioperative symptoms), good (reduction in perioperative symptoms), unchanged (no improvement or worsening of preoperative symptoms).

All patients received at least one preoperative bronchoscopy to rule out a foreign body aspiration, stenosis and/or tumor, and for sputum collection as well as secretion clearance. Whenever feasible, bronchioalveolar lavage was obtained. Chest radiography and high-resolution computed tomography (HRCT) scans prior to surgery were mandatory to classify type, severity, and distribution of bronchiectasis and select patients amenable for resection.

Prior bacterial infection and treatment by appropriate antibiotics, use of bronchodilators, mucolytic agents, and postural drainage as well as a history of intensive respiratory physiotherapy were tracked. All patients were screened for a history of infectious disease (including tuberculosis and/ or NTM), tumor (especially lung cancer and/or metastatic disease of the lung) and hemoptysis.

All included patients received an anatomical lung resection (segmentectomy/lobectomy/bilobectomy) either by minimally invasive (uniportal video-assisted thoracoscopic surgery, VATS) or conventional (lateral muscle sparing thoracotomy) approach and were stratified accordingly.

All resected specimens were subjected to histopathological examination to confirm diagnosis. Postoperative care included respiratory physiotherapy and occasionally antibiotic therapy. All patients received epidural administration of bupivacaine during the operation. Continuous epidural infusion of bupivacaine was used postoperatively until chest tube removal. Metamizol and Paracetamol were predominantly used for postoperative pain control in addition to epidural analgesia. Postoperative air leakage was measured by a digital drainage system (Medela Thopaz ${ }^{\circledR}$, Medela, Baar, Switzerland) and a prolonged fistula was defined as leakage $>20 \mathrm{~mL} / \mathrm{min}$ over a period of $>7$ days.
Perioperative mortality was defined as death within 30 days after surgery or during hospital stay.

\section{Statistical analysis}

Data was collected and analyzed in a standard Microsoft ${ }^{\circledR}$ Excel $^{\circledR} 2010$ spreadsheet and expressed as mean \pm standard deviation (SD) after ascertaining normal distribution via modified Kolmogorov-Smirnov-Test.

\section{Results}

A total of $n=34$ consecutive patients underwent pulmonary resection at our institution due to bronchiectasis between 2016-2020. Mean age was 56.2 \pm 15.1 (range, 17-79) years, $\mathrm{n}=21$ patients $(62 \%)$ were female (Table 1$)$.

Leading symptoms on presentation included persistent cough $(n=29,85 \%)$ and clinical signs of acute infection $(n=17,50 \%)$. Eight patients (24\%) initially presented with acute hemoptysis. Etiologically, tuberculosis $(\mathrm{n}=2,6 \%)$, NTM $(n=2,6 \%)$, secondary aspergilloma $(n=4,12 \%)$ and chronic recurrent infection $(\mathrm{n}=10,29 \%)$ were described. Preoperative BAL was obtained in $n=23$ (68\%) of patients, with $\mathrm{n}=5$ non-representative findings, $\mathrm{n}=6$ cases of granulocytary reaction, $\mathrm{n}=8$ cases of neutrophile alveolitis, $\mathrm{n}=2$ cases of combined neutrophile and eosinophile alveolitis as well as $\mathrm{n}=2$ cases of lymphocytary and eosinophile alveolitis. Microbiological findings include $S$. aureus $(\mathrm{n}=3)$, E. coli $(\mathrm{n}=2)$ and $P$. aeruginosa $(\mathrm{n}=3)$. In $\mathrm{n}=4$, patients' fungal spores ( $\mathrm{n}=3$ with concomitant bacteriological findings) were described.

Main indications for surgical resection were either failure of best medical therapy $(\mathrm{n}=9,26 \%)$ or massive hemoptysis $(\mathrm{n}=4,12 \%)$. In $\mathrm{n}=7$ (21\%; Table 1) cases we found a full blown "destroyed lobe" in our analyzed CT scans. A COPD/bronchiectasis-overlap-syndrome was found in $n=5$ patients that underwent lung volume reduction surgery (LVRS) for severe emphysema, while 6 patients receiving surgical treatment for lung cancer had concomitant bronchiectasis described in their frozen sections.

In $n=23$ cases $(68 \%)$, only the right side was affected (the middle lobe in $\mathrm{n}=13$ cases, $38 \%$ ), the left lung in $\mathrm{n}=9$ cases $(26 \%)$ and both lungs in two cases $(6 \%)$. In $n=21$ patients $(62 \%)$ we performed an anatomical lobectomy, in $\mathrm{n}=2$ cases a bilobectomy and the remaining $\mathrm{n}=11$ patients underwent segmentectomy. Minimally invasive approach (uniportal VATS) was feasible in $n=28$ cases, and only $n=4$ patients undergoing lobectomy as well as those receiving a 
Table 1 Patient characteristics $(n=34)$

\begin{tabular}{|c|c|}
\hline Variables & Value \\
\hline Age (mean $\pm S D)$ & $56.2 \pm 15.0$ \\
\hline Sex (male/female) & $13 / 21(38 / 62)$ \\
\hline \multicolumn{2}{|l|}{ Symptoms, No [\%] } \\
\hline Cough & 29 [85] \\
\hline Clinical signs of acute infection & $17[50]$ \\
\hline Sputum & 19 [56] \\
\hline Hemoptysis & 8 [24] \\
\hline \multicolumn{2}{|l|}{ Causes of bronchiectasis, No [\%] } \\
\hline Chronic recurrent infection & 10 [29] \\
\hline NTM & $2[6]$ \\
\hline Tuberculosis & $2[6]$ \\
\hline Aspergilloma & $4[12]$ \\
\hline COPD overlap & $5[15]$ \\
\hline Unknown & $11[32]$ \\
\hline \multicolumn{2}{|l|}{ Affected lung, No [\%] } \\
\hline Right & $23[68]$ \\
\hline Left & 9 [26] \\
\hline Bilateral & $2[6]$ \\
\hline \multicolumn{2}{|l|}{ Affected lobe, No } \\
\hline Left upper lobe & 6 \\
\hline Left lower lobe & 6 \\
\hline Right upper lobe & 10 \\
\hline Right lower lobe & 6 \\
\hline Middle lobe & 13 \\
\hline Whole left lung & 1 \\
\hline Multiple lobes & 5 \\
\hline \multicolumn{2}{|l|}{ Indications for surgery, No [\%] } \\
\hline Failure of medical treatment & 9 [26] \\
\hline Massive or recurrent hemoptysis & $4[12]$ \\
\hline Destroyed lung & $7[21]$ \\
\hline Sequestration & 0 \\
\hline Bronchial stenosis & 3 [9] \\
\hline Emphysema (Volume reduction) & $5[15]$ \\
\hline Lung cancer & $6[17]$ \\
\hline
\end{tabular}

NTM, non-tuberculous mycobacteria; COPD, chronic obstructive pulmonary disease.
Table 2 Surgical outcome $(\mathrm{n}=34)$

\begin{tabular}{|c|c|}
\hline Postoperative & No [\%] \\
\hline \multicolumn{2}{|l|}{ Surgery } \\
\hline Lobectomy & $21[62]$ \\
\hline Lower lobe & $4[12]$ \\
\hline Upper lobe & 9 [27] \\
\hline Middle lobe & $8[24]$ \\
\hline Segmentectomy & $11[32]$ \\
\hline Bilobectomy & $2[6]$ \\
\hline Pneumonectomy & 0 \\
\hline VATS & $28[82]$ \\
\hline Thorakotomy & $6[18]$ \\
\hline \multicolumn{2}{|l|}{ Complications } \\
\hline Post-operative hemorrhage & 0 \\
\hline Atelectasis & $5[15]$ \\
\hline Persistent air leak $>7$ days & $7[21]$ \\
\hline Empyema & $1[3]$ \\
\hline Wound Infection & 0 \\
\hline Pleural effusion & $7[21]$ \\
\hline Death & $1[3]$ \\
\hline
\end{tabular}

VATS, video-assisted thoracoscopic surgery.

bilobectomy underwent conventional procedures via lateral thoracotomy. Additionally, one of our patients received a "non-intubated VATS lobectomy", a special technique that enables us to forego mechanical ventilation altogether. Results are summarized in Table 2.

Average length of hospital stay was $7.9 \pm 6.3$ days, complications including atelectasis $(\mathrm{n}=5,15 \%)$, pleural effusion $(n=7,21 \%)$, or prolonged air leak $(n=7,21 \%)$, defined as continuous leakage $>20 \mathrm{~mL} / \mathrm{min}>7$ days, were observed in a total of $n=18$ patients $(53 \%)$. Postoperative bleeding or hemoptysis was not observed. Only one patient with complex NTM disease, who developed a pleural empyema after a prolonged fistula, had to undergo a minimally-invasive redo-procedure. One patient died on POD 7 due to acute myocardial infarction (Table 2).

Follow-up was completed in $\mathrm{n}=30$ patients $(88 \%)$ after a mean of $20.4 \pm 13.7$ months. A total of $n=20$ patients $(67 \%)$ considered their own health as "excellent" after surgery, $\mathrm{n}=9(30 \%)$ as "good", and $\mathrm{n}=1(3 \%)$ reported "no change". 


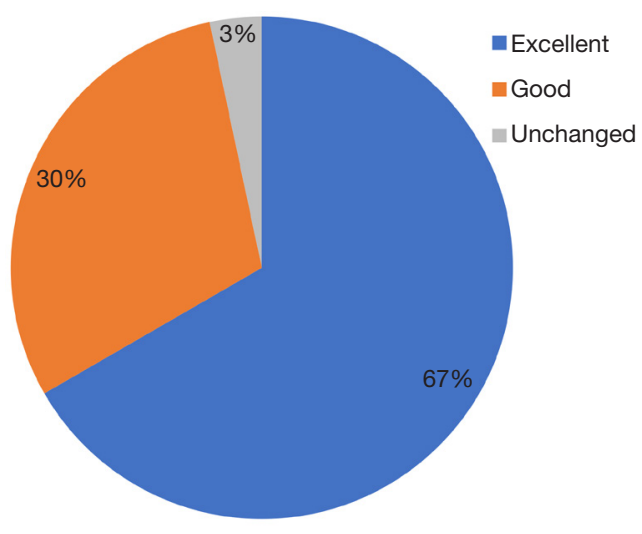

Figure 1 Reported satisfaction after surgery. Follow-up was completed in $\mathrm{n}=30$ patients $(88 \%)$ after a mean of $20.4 \pm$ 13.7 months. A total of $n=20$ patients $(67 \%)$ considered their own health as "excellent" after surgery, $n=9(30 \%)$ as "good", and $n=1$ (3\%) reported "no change".



Figure 2 Preoperative CT scan prior to right lower lobe resection (non-intubated) and subsequent segmentectomy on the left side.

Postoperative outcomes are shown in Figure 1.

\section{Discussion}

Bronchiectasis is characterized by a permanent and mostly irreversible bronchial dilatation and is related to recurring infectious processes, most likely by way of a vicious cycle $(14,16,17)$.

Patients suffer from chronic cough, excessive sputum production and expectoration, a progressive decline in lung function in spite of prolonged antibiotic therapy, and occasionally massive hemoptysis once the disease is advanced (18). Because of continuous improvements in treatment of pulmonary infections and well-established vaccination programs, overall incidence of bronchiectasis requiring dedicated treatment declined over the past decades in most developed countries. Interestingly, a national survey confirms a gradual increase in patients with bronchiectasis in Germany (11), possibly suggesting that bronchiectasis often remains undetected and underappreciated.

Currently, surgical intervention is mainly reserved for patients with focal disease with persistent symptoms despite optimal medical management and those presenting with acute hemoptysis.

By far and large, the most common symptom in bronchiectasis is chronic, productive cough $(16,19)$, which was observed in $85 \%$ of our patients, often accompanied by copious amounts of expectorations (56\% in our collective). Even though we had a high rate of patients presenting with hemoptysis (24\%) presenting clinical symptoms were well in line with most other tertiary outfits (15). Due to tenacious investigations by our referring pneumologists, we were able to identify a cause for bronchiectasis in $53 \%$ of all patients admitted for surgery, which is slightly beyond results obtained by other groups (15): according to published data, underlying pathologies are discovered in less than $40 \%$ admitted for treatment $(8,16)$. This may be attributable to our systematic work-up: As per protocol, all our patients underwent lung function testing, standard X-ray and HRCT. Even a $10-\mathrm{mm}$ slice-thickness HRCT is sufficient to determine distribution and severity of disease with a sensitivity of $66 \%$ and a specificity of $92 \%$ (20). Additional bronchoscopy was always performed as well to rule out foreign bodies or endobronchial masses that may cause bronchiectasis. In-depth examination of our patients past medical history confirmed documented chronic infectious disease in more than $50 \%$ of cases. As a regional referral center for NTM-disease, which can be associated with bronchiectasis, we treated a certain number of patients with this pathology. NTM can be divided into two subgroups radiologically, a fibrocavitary and a nodular/bronchiectatic type (21), with the latter being almost impossible to discern from post-infectious bronchiectasis colonized by NTM (22). Despite overlapping clinical features with conventional non-CF-bronchiectasis, NTM-management is particularly complex and a surgical approach may be viable to induce treatment success through removal of 
septic lung portion allowing drug therapy discontinuation, symptomatic treatment of hemoptysis, or partial resection in advanced cases to slow down overall disease progression (Figure 2) (23). Our 2 included cases of NTM were referred to surgery for complete resection of all infectious sites, in one case by bilateral procedures.

Recent data suggests that COPD and bronchiectasis may coexist in $20-60 \%$ of cases (24), and in our series we found evidence of bronchiectasis in 5 patients undergoing surgical LVRS for severe bullous emphysema. As COPD/ bronchiectasis overlap appears to lead to increased symptoms and a worse prognosis compared to COPD or bronchiectasis alone $(6,25)$, a reasonable fraction of patients with severe COPD, especially those considered suitable for surgical treatment, can be expected to have concomitant bronchiectasis. Unfortunately, at this point no clear-cut evidence or specific biomarkers linking both diseases exist (6), making this a mostly anecdotal observation. Given the known association between COPD and lung cancer (26), either as a driving factor or by having similar risk profiles like genetic predisposition or smoking habits, a further overlap with bronchiectasis is likely. A nationwide cohort-study in Taiwan demonstrated a 2.36fold increased risk for lung cancer in bronchiectasispatients after adjusting for age, sex and co-morbidities (27), even though evidence effectively linking those three diseases still is sparse. In our collective, $n=6$ patients who underwent curative lung cancer surgery were confirmed with bronchiectasis, suggesting that a further investigation of COPD/Lung cancer/bronchiectasis-overlap may be warranted, and surgical specimens obtained in LVRS or cancer surgery should routinely be screened for presence of bronchiectasis.

Generally, bronchiectasis affects dependent portions of the lung, which includes posterior basal portions of the lower lobes, middle lobe and lingula. 32 out of our 34 patients had a unilateral affliction, which may be due to a selection bias, as only patients potentially amenable for complete and radical surgical treatment were discussed in our MDT-conferences.

Failure of best medical therapy, including repeated antibiotic treatment courses, was the most commonly reported indication for surgical intervention (15). Only a small percentage of patients presented with significant hemoptysis or lung abscess. Similar to other series, the majority of our patients had a history of recurrent respiratory infections, which emphasizes the importance of appropriate medical treatment and MDT-management.
Targeted antibiotic therapy, bronchodilators, and physiotherapy were part of bronchiectasis management in all patients prior to resection. Interestingly, recently Mariani et al. reported a series of 44 patients scheduled for surgical resection in bronchiectasis, and discovered a positive BAL culture as predictor for postoperative complications (28). In our collective, we found that $\mathrm{n}=7$ out of 20 patients having postoperative complications had positive cultures as well. This includes a case with empyema (NTM), $n=3$ patients with positive cultures for s. aureus developing secondary pleural effusion $(n=1)$ or atelectasis $(n=2), 2$ cases of atelectasis in confirmed $\mathrm{E}$. coli and one atelectasis in Pseudomonas aeruginosa. As only a fraction of our patients underwent preoperative BAL, these findings have to be considered circumstantial, but warrant further investigation.

As confirmed by a recent meta-analysis, that included 127 patients in 3 studies undergoing surgery for bronchiectasis in developed countries since 2000, resection for non-CF-bronchiectasis in western countries remains the exception (29-31). Interestingly, even though all 3 groups agreed on anatomical lung resection as therapy of choice for all patients, only Giovannetti and co-workers detailed their surgical approach (31). In their study, the majority of patients underwent a limited posterolateral thoracotomy, and a smaller fraction received minimally-invasive treatment. VATS has become the standard approach for anatomic lung resection and replaced conventional thoracotomy over the past decade. It is associated with reduced postoperative pain and increased quality of life $(32,33)$ as well as shortened length of hospital stay (34), and represents an ideal method for older and multimorbid patients otherwise not amenable for conventional surgery. It is better tolerated owing to less chest wall injury and better preservation of postoperative lung function (35).

Severe adhesions to the chest wall, the hilum, and in the fissure, always to be expected in inflammatory disease, may prove a limiting factor for VATS. This prompts other groups to generally proceed by thoracotomy (28). However, over $80 \%$ of patients in our population (including $n=11$ VATS-segmentectomies and $\mathrm{n}=17$ VATS-lobectomies) were operated on successfully by minimally invasive approach, thus demonstrating the feasibility in this population.

In conclusion, with an overall low morbidity and mortality, minimally invasive anatomical lung resection still should be considered in treating selected patients with non-CF-bronchiectasis in Western Europe, and further investigation of a possible COPD/Lung cancer/ bronchiectasis-overlap may be warranted. 


\section{Acknowledgments}

Funding: None.

\section{Footnote}

Reporting Checklist: The authors have completed the STROBE reporting checklist. Available at https://dx.doi. org/10.21037/jtd-21-879

Data Sharing Statement: Available at https://dx.doi. org/10.21037/jtd-21-879

Peer Review File: Available at https://dx.doi.org/10.21037/ jtd-21-879

Conflicts of Interest: All authors have completed the ICMJE uniform disclosure form (available at https://dx.doi. org/10.21037/jtd-21-879). The authors have no conflicts of interest to declare.

Ethical statement: The authors are accountable for all aspects of the work in ensuring that questions related to the accuracy or integrity of any part of the work are appropriately investigated and resolved. The study was conducted in accordance with the Declaration of Helsinki (as revised in 2013). The study was approved by institutional ethics board of the Hannover Medical School (NO.: 9789_ B0_K_2021) and individual consent for this retrospective analysis was waived.

Open Access Statement: This is an Open Access article distributed in accordance with the Creative Commons Attribution-NonCommercial-NoDerivs 4.0 International License (CC BY-NC-ND 4.0), which permits the noncommercial replication and distribution of the article with the strict proviso that no changes or edits are made and the original work is properly cited (including links to both the formal publication through the relevant DOI and the license). See: https://creativecommons.org/licenses/by-nc-nd/4.0/.

\section{References}

1. Chiarenza A, Esposto Ultimo L, Falsaperla D, et al. Chest imaging using signs, symbols, and naturalistic images: a practical guide for radiologists and non-radiologists. Insights Imaging 2019;10:114.

2. Bird K, Memon J. Bronchiectasis. Treasure Island (FL):
StatPearls Publishing LLC., 2021.

3. McShane PJ, Naureckas ET, Tino G, et al. Non-cystic fibrosis bronchiectasis. Am J Respir Crit Care Med 2013;188:647-56.

4. Poeta M, Maglione M, Borrelli M, et al. Non-cystic fibrosis bronchiectasis in children and adolescents: Neglected and emerging issues. Pediatr Neonatol 2020;61:255-62.

5. Chang AB, Bush A, Grimwood K. Bronchiectasis in children: diagnosis and treatment. Lancet 2018;392:866-79.

6. Polverino E, Dimakou K, Hurst J, et al. The overlap between bronchiectasis and chronic airway diseases: state of the art and future directions. Eur Respir J 2018;52:1800328.

7. Balkissoon R. Journal Club - Bronchiectasis/COPD Overlap: Syndrome Versus Treatable Trait? Chronic Obstr Pulm Dis 2019;6:193-9.

8. Cohen M, Sahn SA. Bronchiectasis in systemic diseases. Chest 1999;116:1063-74.

9. Ringshausen FC, de Roux A, Diel R, et al. Bronchiectasis in Germany: a population-based estimation of disease prevalence. Eur Respir J 2015;46:1805-7.

10. Monteagudo M, Rodríguez-Blanco T, Barrecheguren $M$, et al. Prevalence and incidence of bronchiectasis in Catalonia, Spain: A population-based study. Respir Med 2016;121:26-31.

11. Ringshausen FC, Rademacher J, Pink I, et al. Increasing bronchiectasis prevalence in Germany, 20092017: a population-based cohort study. Eur Respir J 2019;54:1900499.

12. Doğan R, Alp M, Kaya S, et al. Surgical treatment of bronchiectasis: a collective review of 487 cases. Thorac Cardiovasc Surg 1989;37:183-6.

13. Halezeroglu S, Keles M, Uysal A, et al. Factors affecting postoperative morbidity and mortality in destroyed lung. Ann Thorac Surg 1997;64:1635-8.

14. King PT. The pathophysiology of bronchiectasis. Int J Chron Obstruct Pulmon Dis 2009;4:411-9.

15. Eren S, Esme H, Avci A. Risk factors affecting outcome and morbidity in the surgical management of bronchiectasis. J Thorac Cardiovasc Surg 2007;134:392-8.

16. Barker AF. Bronchiectasis. N Engl J Med 2002;346:1383-93.

17. Cole PJ. Inflammation: a two-edged sword--the model of bronchiectasis. Eur J Respir Dis Suppl 1986;147:6-15.

18. Baydarian M, Walter RN. Bronchiectasis: introduction, etiology, and clinical features. Dis Mon 2008;54:516-26.

19. Nicotra MB, Rivera M, Dale AM, et al. Clinical, 
pathophysiologic, and microbiologic characterization of bronchiectasis in an aging cohort. Chest 1995;108:955-61.

20. Cooke JC, Currie DC, Morgan AD, et al. Role of computed tomography in diagnosis of bronchiectasis. Thorax 1987;42:272-7.

21. Griffith DE, Aksamit T, Brown-Elliott BA, et al. An official ATS/IDSA statement: diagnosis, treatment, and prevention of nontuberculous mycobacterial diseases. Am J Respir Crit Care Med 2007;175:367-416.

22. Hiramatsu M, Shiraishi Y. Surgical management of non-cystic fibrosis bronchiectasis. J Thorac Dis 2018;10:S3436-45.

23. Mitchell JD. Surgical approach to pulmonary nontuberculous mycobacterial infections. Clin Chest Med 2015;36:117-22.

24. Du Q, Jin J, Liu X, et al. Bronchiectasis as a Comorbidity of Chronic Obstructive Pulmonary Disease: A Systematic Review and Meta-Analysis. PLoS One 2016;11:e0150532.

25. Martinez-Garcia MA, Miravitlles M. Bronchiectasis in COPD patients: more than a comorbidity? Int J Chron Obstruct Pulmon Dis 2017;12:1401-11.

26. Durham AL, Adcock IM. The relationship between COPD and lung cancer. Lung Cancer 2015;90:121-7.

27. Chung WS, Lin CL, Hsu WH, et al. Increased risk of lung cancer among patients with bronchiectasis: a nationwide cohort study. QJM 2016;109:17-25.

28. Mariani AW, Vallilo CC, de Albuquerque ALP, et al. Preoperative evaluation for lung resection in patients with bronchiectasis: should we rely on standard lung function

Cite this article as: Selman A, Merhej H, Nakagiri T, Zinne N, Goecke T, Haverich A, Zardo P. Surgical treatment of non-cystic fibrosis bronchiectasis in Central Europe. J Thorac Dis 2021;13(10):5843-5850. doi: 10.21037/jtd-21-879 evaluation? Eur J Cardiothorac Surg 2021;59:1272-8.

29. Fan LC, Liang S, Lu HW, et al. Efficiency and safety of surgical intervention to patients with Non-Cystic Fibrosis bronchiectasis: a meta-analysis. Sci Rep 2015;5:17382.

30. Hiramatsu M, Shiraishi Y, Nakajima Y, et al. Risk factors that affect the surgical outcome in the management of focal bronchiectasis in a developed country. Ann Thorac Surg 2012;93:245-50.

31. Giovannetti R, Alifano M, Stefani A, et al. Surgical treatment of bronchiectasis: early and long-term results. Interact Cardiovasc Thorac Surg 2008;7:609-12.

32. Pu Q, Ma L, Mei J, et al. Video-assisted thoracoscopic surgery versus posterolateral thoracotomy lobectomy: A more patient-friendly approach on postoperative pain, pulmonary function and shoulder function. Thorac Cancer 2013;4:84-9.

33. Bendixen M, Jørgensen OD, Kronborg C, et al. Postoperative pain and quality of life after lobectomy via video-assisted thoracoscopic surgery or anterolateral thoracotomy for early stage lung cancer: a randomised controlled trial. Lancet Oncol 2016;17:836-44.

34. Zhang P, Zhang F, Jiang S, et al. Video-assisted thoracic surgery for bronchiectasis. Ann Thorac Surg 2011;91:239-43.

35. Usuda K, Maeda S, Motomo N, et al. Pulmonary Function After Lobectomy: Video-Assisted Thoracoscopic Surgery Versus Muscle-Sparing Mini-thoracotomy. Indian J Surg 2017;79:504-9. 